\title{
Severe Hypoglycemia Contributing to Cognitive Dysfunction in Diabetic Mice is Associated with Pericyte and Blood-Brain Barrier Dysfunction
}

Lu Lin

Fujian Medical University Union Hospital

Lishan Huang

Fujian Medical University

Lijing Wang

Fujian Medical University Union Hospital

Yubin Wu

Fujian Medical University Union Hospital

Zhou Chen

Fujian Medical University Union Hospital

Libin Liu ( $\square$ libinliu@fjmu.edu.cn )

Fujian Medical University Union Hospital

\section{Research}

Keywords: Severe hypoglycemia, Diabetes mellitus, Cognitive dysfunction, Pericyte, Blood-brain barrier

Posted Date: September 22nd, 2021

DOI: https://doi.org/10.21203/rs.3.rs-903076/v1

License: (c) (1) This work is licensed under a Creative Commons Attribution 4.0 International License.

Read Full License

Version of Record: A version of this preprint was published at Frontiers in Aging Neuroscience on November 26th, 2021. See the published version at https://doi.org/10.3389/fnagi.2021.775244. 


\section{Abstract}

Background: Severe hypoglycemia can cause cognitive impairment in diabetic patients, but the underlying molecular mechanism remains unclear.

Objective: To assess the effect of severe hypoglycemia on cognitive function in diabetic mice to clarify the relationship between the mechanism and dysfunction of pericytes and the blood-brain barrier (BBB).

Method: We established type 1 diabetes mellitus in 80 male C57BL/6J mice by intraperitoneal injection of streptozotocin $(180 \mathrm{mg} / \mathrm{kg})$. Further abdominal injection of short-acting insulin induced severe hypoglycemia. The mice were divided into normal, diabetes, and diabetic + severe hypoglycemia groups, and their blood glucose and general weight index were examined. Pericyte and BBB morphology and function were detected by histological and western blot analyses, BBB permeability was detected by Evans blue staining, and cognitive function was detected with the Morris water maze.

Results: Severe hypoglycemia aggravated the histological damage, BBB damage, brain edema, and pericyte loss in the diabetic mice. It also reduced the expression of the BBB tight junction proteins occludin and claudin-5, the expression of the pericyte-specific markers PDGFR- $\beta$ (platelet-derived growth factor receptor- $\beta$ ) and $\alpha-S M A$, and increased the expression of the inflammatory factor MMP9. At the same time, diabetic mice with severe hypoglycemia had significantly reduced cognitive function.

Conclusion: Severe hypoglycemia leads to cognitive dysfunction in diabetic mice, and its possible mechanism is related to pericyte dysfunction and BBB destruction.

\section{Introduction}

According to the latest data [1], it is estimated that by 2045 , the number of diabetic patients will reach 700.2 million, and the prevalence of diabetes is rapidly rising. Hypoglycemia, hyperglycemia, and high blood glucose fluctuations are the three main characteristics in diabetes management [2]. One important therapeutic approach for treating diabetes is maintaining normal blood glucose levels and reducing the occurrence of hypoglycemia. Cognitive function refers to all aspects of thinking and intellectual activities, and is fundamental to everyday activities. Cognitive dysfunction refers to different degrees of cognitive impairment stemming from various causes, and includes mild cognitive dysfunction and dementia [3]. Even minor changes in cognitive function potentially affect a person's ability to achieve the best functional state, while severe cognitive dysfunction can severely reduce the patient's quality of life and can even be life-threatening. Although epidemiological evidence shows that diabetes is closely related to cognitive dysfunction [4], this result remains controversial, and the exact mechanism is unknown. Many molecular and pathological consequences of diabetes overlap with factors that may lead to dementia [5], such as changes in insulin signaling, advanced glycosylation products, and chronic low-grade inflammation, all of which are common potential mechanisms for neurological degeneration [6]. However, an increasing number of studies have suggested that hypoglycemia plays an important role in the pathogenesis of cognitive dysfunction in diabetic patients [7, 8]. Short-term mild hypoglycemia can cause 
reversible cognitive function impairment, while sustained or severe hypoglycemia can cause permanent neuronal damage, which further damages the brain structure and leads to changes in cognitive function [9]. However, the pathogenesis of cognitive dysfunction caused by severe hypoglycemia remains poorly understood.

Alzheimer disease (AD) is the most common form of dementia, and there are no therapies to slow its progression or delay its onset. Regarding $A D$ pathogenesis, amyloid- $\beta$ protein $(A \beta)$ and tau protein immediately come to mind. With the successive failure of drugs targeting both proteins, it has become clear that the deposition of $A \beta$ protein and tau protein may not clarify all $A D$ pathogenesis [10]. The Zlokovic team showed that AD patients have increased blood-brain barrier (BBB) permeability, and detected the level of soluble platelet-derived growth factor receptor- $\beta$ (PDGFR- $\beta$ ) (a biomarker of pericyte damage) in cerebrospinal fluid [11]. It is worth mentioning that a recent study using a rat model of cerebral small vessel disease showed that restoring BBB integrity by infusing pericyte precursor cells improved cognitive function [12]. In summary, pericyte dysfunction, characterized by BBB destruction, may be involved in the pathological process of cognitive dysfunction-related diseases. Epidemiological and animal studies have found that diabetic patients have enhanced BBB permeability, which is related to the loss of pericyte function [13, 14]. However, Maarja et al. [15] found that long-term systemic hyperglycemia alone did not cause enhanced BBB permeability and impaired pericellular function in Ins2AKITA genetic diabetic mice. The authors suggested that, in addition to hyperglycemia, other features of diabetes, such as the hyperglycemia-hypoglycemia cycle, should also be considered as possible causative factors. Hypoglycemic coma significantly increased BBB permeability in the cerebral cortex, cerebellum, and brainstem regions [16]. In addition, acute severe hypoglycemia-induced inflammation may destroy the BBB by reducing the expression of tight junction proteins [17]. However, the relationship and mechanism between hypoglycemia and BBB are unclear.

In recent years, the relationship between impaired BBB/pericyte function and cognitive dysfunction has attracted increased attention. As the chain of evidence from the above studies continues to be updated, it is reasonable to speculate that severe hypoglycemia plays an important role in the pathogenesis of cognitive dysfunction in diabetic patients and that this role is associated with impaired BBB/pericyte function. Nevertheless, there is no research on the issue.

In the present study, we investigated the effect of severe hypoglycemia on cognitive function in diabetic mice to clarify the relationship between decreased cognitive function and pericyte and BBB dysfunction due to severe hypoglycemia. Our findings will aid the elucidation of the relationship and related mechanisms between severe hypoglycemia and cognitive dysfunction.

\section{Materials And Methods}

\subsection{Experimental animal experimental design}


Male C57BL/6J mice ( $\mathrm{n}=80,20-25 \mathrm{~g})$ were purchased from Zhejiang Experimental Animal Center. All experimental steps were conducted in accordance with the Animal Care and Use Guidelines of the China Experimental Society and were approved by the Fujian Animal Research Ethics Commission (Approval No. FJMU IACUC 2018-060). The animals were kept under controlled temperature and humidity in a 12-h dark-light cycle (6:00 h, lights on; 18:00 h, lights off), and food and water were freely available. After 1 week of adaptation, the mice were divided into three groups (20 mice per group) using the random number method: normal group (NC), diabetic group (DM), and diabetic + severe hypoglycemia group (DH). The DM and DH groups were treated with a single intraperitoneal (ip) injection of streptozotocin (STZ; S0130; Sigma, St. Louis, MO, USA) dissolved in $1 \%(\mathrm{w} / \mathrm{v})$ solution of $0.1 \mathrm{M}$ citrate buffer $(\mathrm{pH} 4.2-4.5)$ at a dose of $150 \mathrm{mg} / \mathrm{kg}$. The NC group was injected with an equal amount of citrate buffer. On day 3 after STZ injection, random blood glucose levels were measured using a FreeStyle glucometer (Abbott, Berkshire, UK) to determine whether diabetes had been successfully induced. Mice with three consecutive randomized blood glucose levels of $\geq 16.7 \mathrm{mmol} / \mathrm{L}$ and diabetic behavior changes (increased dietary intake, increased urination, weight loss) were considered successful in type 1 diabetes mimicry. Failed mice received a second STZ injection, and blood glucose testing was repeated.

The diabetic mouse severe hypoglycemia model was established referring to the previous model of our group [18]. After 8-h fast overnight, the DH group received injections of short-acting insulin (Wanbang, Jiangsu, China, dose $15 \mathrm{mU} / \mathrm{g}$ ip); the $\mathrm{NC}$ and DM groups received equal volumes of saline under the same conditions. Tail vein glucose was assessed every $30 \mathrm{~min}$ to ensure that the mice maintained low blood glucose levels ( $<2.0 \mathrm{mmol} / \mathrm{L}$ ) over $90 \mathrm{~min}$. To terminate hypoglycemia, the mice were allowed to feed or were injected with glucose (1 mg/kg ip). One mouse in the DM group and three mice in the DH group died after the modelling.

\subsection{Histologic analyses}

\subsubsection{Hematoxylin-eosin (HE) staining}

After 24-h severe hypoglycemia modeling, 46 mice from all three groups was killed for histological testing; the remaining mice $(n=30)$ underwent cognitive function testing in a Morris water maze test on day 8 of severe hypoglycemia (Fig. 1). Morris water maze test were done after 7 days to allow the mice to recover sufficiently and to exclude factors such as lethargy and bad mood due to hypoglycemia from affecting the effect of the Morris water maze test [9].

The mice were anesthetized with $2 \%$ sodium pentobarbital $(2 \mathrm{ml} / \mathrm{kg}$ ip), and the brains were quickly removed on ice, fixed in $4 \%$ paraformaldehyde for $48 \mathrm{~h}$, and embedded in paraffin. The paraffin sections were dewaxed to water, sequentially stained with hematoxylin, stained with eosin, sealed by dehydration, and examined under a microscope. The HE-stained hippocampal neurons were observed, and their images were acquired for analysis.

\subsubsection{Immunohistochemistry and immunofluorescence staining}


PDGFR- $\beta$ expression levels in the mouse brain tissue were detected with immunohistochemistry.

Paraformaldehyde-fixed, paraffin-embedded specimens were removed, autoclaved, heat-treated in citrate saline buffer to extract antigens, and incubated with rabbit antibody (anti-PDGFR- $\beta, 1: 200, a b 32570$, Abcam) overnight at $4^{\circ} \mathrm{C}$. All steps were performed according to the manufacturer's protocols. Positive staining results were brown. Images of the immunohistochemically stained sections were analyzed with Image-Pro Plus 6.0 (Media Cybernetics, Silver Spring, MD, USA).

PDGFR- $\beta$ expression levels in the mouse brain tissue were examined with immunofluorescence staining. Paraffin sections were dewaxed to water, antigen-repaired, and serum-blocked. Then, the blocking solution was gently shaken off, primary antibody (anti-PDGFR- $\beta, 1: 200$, ab32570, Abcam) was added dropwise to the sections, and the sections were incubated flat in a wet box overnight at $4^{\circ} \mathrm{C}$. Then, the samples were incubated with secondary antibody before the nuclei were restained with DAPI. Next, the autofluorescence of the tissue was quenched, the sections were sealed, and the images were obtained under a fluorescent microscope. The DAPI-stained nuclei were blue under UV excitation and positively expressed as luciferin-labeled green fluorescence.

\subsubsection{Transmission electron microscope examination}

Fresh mouse brain hippocampal tissue $\left(1 \mathrm{~mm}^{3}\right)$ was obtained within 1-3 min to minimize mechanical damage such as pulling, contusion, and extrusion. The brain tissue blocks were transferred to Eppendorf tubes with fresh electrodense fixing fluid and stored and transported at $4^{\circ} \mathrm{C}$. The samples were rinsed three times with $0.1 \mathrm{M}$ phosphate buffer $(\mathrm{pH} 7.4)$ for $15 \mathrm{~min}$ each time. Then, they were fixed, dehydrated at room temperature, and infiltration-embedded, and the embedding board was placed in a $60^{\circ} \mathrm{C}$ oven for 48-h polymerization. Subsequently, ultra-thin slices were obtained and stained. The sections were observed under a transmission electron microscope, and the acquired images were analyzed using Image-Pro Plus 6.0 (Media Cybernetics).

\subsection{Evaluation of BBB permeability}

A 2\% concentration of Evans blue solution (E2129, Sigma) was prepared in advance with saline and injected through the mouse tail vein at a dose of $4 \mathrm{ml} / \mathrm{kg}$ at $18 \mathrm{~h}$ after severe hypoglycemia. After 6-h in vivo circulation, the mice were anesthetized and perfused with phosphate-buffered saline through the heart to remove residual Evans blue from the blood vessels. Then, the heads were removed, and the brain tissue was photographed with a Nikon D750 camera (Tokyo, Japan). Next, the brains were homogenized in $50 \%$ trichloroacetic acid solution (T5159, Sigma) and centrifuged at 20,000 $\times g$ for 20 min. The supernatant was collected and mixed with anhydrous ethanol (1:3). Evans blue extravasation was quantified by measuring the fluorescence intensity (excitation wavelength, $620 \mathrm{~nm}$; emission wavelength, $680 \mathrm{~nm}$ ). The amount of Evans blue dye was calculated using a standard curve and expressed as $\mu \mathrm{g} / \mathrm{g}$ brain tissue.

\subsection{Measurement of brain water content}


Cortical water content is a sensitive indicator of brain edema and can be used for assessing hypoglycemia-induced brain edema and disruption of BBB function. After the mice had been anesthetized with isoflurane and killed, the brains were quickly removed, and the cerebellum and olfactory bulb were discarded. The brain tissue was immediately weighed to obtain the wet weight $(W)$, then oven-dried at $65^{\circ} \mathrm{C}$ for $72 \mathrm{~h}$ and re-weighed to obtain the dry weight (D). The brain water content (\%) was calculated as $(\mathrm{W}-\mathrm{D}) / \mathrm{W} \times 100 \%$.

\subsection{Western blot analysis}

After the remaining mice in each group had been euthanized, the brains were quickly removed from the ice surface, the hippocampus on both sides was separated and placed in lyophilization tubes and then quickly immersed in liquid nitrogen, the tissues were rapidly frozen and then immediately transferred to an $-80^{\circ} \mathrm{C}$ refrigerator for storage, and set aside for subsequent testing. The protein concentration in the supernatant was detected using a bicinchoninic acid assay kit (Beyotime, Shanghai, China) according to the manufacturer's instructions. Proteins were separated using $10 \%$ sodium dodecyl sulfatepolyacrylamide gel electrophoresis. The primary antibodies used were as follows: PDGFR- $\beta$ (1:1000, ab32570, Abcam), a-SMA (1:1000, ab7871, Abcam), occludin (1:1000, ab236127, Abcam), claudin-5 (1:1000, ab131259, Abcam), MMP9 (1:1000, GB11132, Servicebio), $\beta$-actin (1:2000, ab8226, Abcam), and GAPDH (1:2000, ab8245, Abcam). The membranes were then incubated with horseradish peroxidaseconjugated anti-rabbit or anti-mouse IgG (1:3000; Cell Signaling Technology) for $1 \mathrm{~h}$ at room temperature and visualized by exposure to Kodak film after detection with chemiluminescent reagent (Millipore). The strip density was quantified with ImageJ analysis software.

\subsection{Grip strength test}

To confirm that severe hypoglycemia did not impair muscle strength or flexibility, the mice underwent grip strength tests three times before the water maze test; the time spent hanging from a wire was recorded.

\subsection{Morris water maze}

The Morris water maze consists of a 120-cm diameter, $30-\mathrm{cm}$ high cylinder with a controlled water temperature of around $22^{\circ} \mathrm{C}$. One week after severe hypoglycemia, the swimming trajectories of the mice ( $n=10$ per group) were analyzed by a camera tracking software system. In the hidden platform experiment, the platform with a diameter of about $7 \mathrm{~cm}$ was located $1.5 \mathrm{~cm}$ below the water surface. The mice were trained to swim for five consecutive days, four times a day. The quadrant order of each daily water entry was determined based on semi-random distribution numbers. The maximum duration of each training was $60 \mathrm{~s}$. If the mice could not find the hidden platform within the specified time, the latency period was recorded as $60 \mathrm{~s}$, and they were guided to stay and rest on the platform for $15 \mathrm{~s}$. Swimming distance, speed, and trajectory were recorded by the system camera. The platform was removed $24 \mathrm{~h}$ after the last day of hidden platform training. The mice entered the water from the opposite quadrant to the original platform, and the camera tracking system recorded the time taken, the number of times the mice crossed the original platform, and the swimming path in each quadrant within $60 \mathrm{~s}$.

\subsection{Statistical analysis}


All experimental data were statistically analyzed using SPSS 25.0 statistical software. Data from the hidden platform experiment were analyzed using repeated-measure two-way analysis of variance (ANOVA). The chi-square of the experimentally normally distributed measures was tested using Levene's test (0.05). The sample means of each group that met the chi-square test requirements were compared using one-way ANOVA and LDS tests; the Kruskal-Wallis $\mathrm{H}$ test was used for data that did not meet the chi-square test requirements. Experimental data are expressed as the mean $\pm \mathrm{SEM}$, and $P<0.05$ represents statistically significant differences.

\section{Results}

\subsection{Physical and biochemical parameters}

Compared with the NC group, mice in the DM and DH groups had significantly higher blood glucose at day 3 after STZ injection (Fig. 2A). DM and DH mice had significantly decreased body weight from baseline (Fig. 2B). In addition, both DM and DH mice also showed obvious symptoms of polyuria, polydipsia, and polydipsia, indicating successful establishment of the type 1 diabetes model. Figure $2 \mathrm{C}$ shows that the $\mathrm{DH}$ group started ip insulin injection at 0 min to induce the occurrence of severe hypoglycemia (red arrow), and that blood glucose dropped significantly $30 \mathrm{~min}$ after the injection. During severe hypoglycemia, the glucose levels in the $\mathrm{DH}$ mice were maintained at $<2.0 \mathrm{mmol} / \mathrm{L}$ and the status lasted for $90 \mathrm{~min}$ (60-150 min). The hypoglycemic episode was terminated by ip injection of glucose at 150 min, and free feeding was allowed (black arrow). The next day, tail vein blood glucose was measured in each group, and blood glucose in the $\mathrm{DH}$ group returned to the level before severe hypoglycemia (Fig. 2A).

\subsection{Severe hypoglycemia in diabetic conditions caused histological alterations in hippocampal neurons}

We used HE staining to assess the histological alterations of the mouse hippocampal neurons. The NC group had regular hippocampal neuron structure, and there were 4-5 layers of pyramidal cells neatly and closely arranged, surrounded by red cytoplasm at the periphery. A large and round nucleus could be observed in the vertebral cells, and 1-2 nucleoli could be clearly seen in the nucleus (Fig. 3A). In contrast, the DH group had disorganized hippocampal pyramidal cells, with shrunken cytosomes and shrunken or absent nuclei (Fig. 4C). The DM group had some degree of damage as compared with the DH group, but the morphological condition of the cone cells was better than that of the DH group (Fig. 4B).

\subsection{Severe hypoglycemia caused BBB destruction and pericyte loss}

\subsubsection{Immunohistochemical and immunofluorescence staining}


We used immunohistochemistry and immunofluorescence staining to observe the expression of the pericyte-specific antibody PDGFR- $\beta$ in mouse brain tissue. Immunohistochemistry (Fig. 4A, B) showed that the DH group had significantly reduced PDGFR- $\beta$ expression compared to the NC group $(P<0.05)$, while no significant difference was seen in the DM group $(P>0.05)$. Immunofluorescence staining (Fig. 4C) showed that PDGFR- $\beta$ protein was stably expressed along the surface of intracerebral microvessels in the NC group. Compared with the NC group, the DM and DH groups had decreased PDGFR- $\beta$ protein expression on the surface of the brain microvessels, and the decrease in the $\mathrm{DH}$ group was the most obvious. The mean optical density of PDGFR- $\beta$ positivity was quantified and was significantly lower in the DM and DH groups compared to the NC group (NC, $0.069 \pm 0.009$ vs. DM, 0.054 $\pm 0.002, P<0.05$; NC, $0.069 \pm 0.009$ vs. $\mathrm{DH}, 0.047 \pm 0.009$, $P<0.05$; Fig. 4D).

\subsubsection{Transmission electron microscopy}

We used transmission electron microscopy to evaluate the effects of severe hypoglycemia on BBB structure and pericyte morphology in the mice. The NC group had normal BBB, and the endothelial cells (EC) were flat and long shuttle-shaped with long nuclei, with intact nuclear membranes and normal perinuclear gaps (Fig. 5). The mitochondrial membranes were intact, with normal-colored intramembrane matrix and the presence of cristae. The rough endoplasmic reticulum (RER) was not dilated, ribosome attachment was visible on the surface, and the Golgi apparatus was not proliferated or hypertrophied. Myelin-like structures were seen in the capillary lumen (Cap), and the basement membrane (BM) was continuous, intact, and of uniform thickness. There was a high number of intercellular tight junctions (TJ), and narrow intercellular space. Under further magnification, the pericytes were not edematous, the stroma was uniform, the nuclei were irregularly shaped, the nuclear membrane was intact, and the perinuclear gaps were normal. Compared with the NC group, the DH group showed severe BBB damage, severe EC edema, severe mitochondrial swelling, intramembrane matrix lysis, and cristae reduction and disappearance. The RER was markedly dilated and locally degranulated. The Cap was markedly atrophied and collapsed. The BM structure was blurred. The intercellular TJ were lost and the cell gaps were narrowed. The astrocyte footplate was markedly edematous, with sparse stroma. Further magnification revealed severe pericyte edema and intracellular low-electron density edema. The nuclei were irregularly shaped, the perinuclear gap was widened, and the heterochromatin boundary was set. Compared with the DH group, the DM group had milder impairment of the above indicators and mild BBB and pericyte impairment.

\subsection{Severe hypoglycemia aggravated BBB Evans blue leakage and brain edema}

We assessed the effect of severe hypoglycemia on BBB permeability in the mice using Evans blue, and quantified the Evans blue extravasation and brain water content in mice under severe hypoglycemia. The $\mathrm{NC}$ mouse brains were not stained with Evans blue, while that of the DM group were slightly blue, and the DH group had obvious penetration of Evans blue (Fig. 6A). Quantification of Evans blue in the brain revealed that the $\mathrm{DH}$ group had significantly higher Evans blue content than the other two groups (NC vs. 
$\mathrm{DH}, P<0.01$, DM vs. DH, $P<0.05$; Fig. 6B), suggesting that severe hypoglycemia significantly aggravates BBB disruption in diabetic mice. The water content in the brain tissue was calculated by weighing the weight of the brain tissue before and after drying. The results showed that the $\mathrm{DH}$ group had significantly higher brain water content compared with the NC group $(P<0.01)$, suggesting that severe hypoglycemia aggravates brain edema in diabetic mice.

\subsection{Occludin, claudin-5, MMP9, PDGFR- $\beta$, and $\alpha-S M A$ expression after severe hypoglycemia}

The expression levels of the BBB TJ proteins occludin and claudin-5, the pericyte-specific proteins PDGFR$\beta$ and a-SMA, and the pericyte injury-related protein MMP9 in the mouse hippocampus were evaluated with western blotting. Compared with the NC group, the DM and DH groups had reduced occludin and claudin-5, which were significantly decreased in the $\mathrm{DH}$ group (NC vs. $\mathrm{DM}, P<0.05$, NC vs. $\mathrm{DH}, P<0.01$; Fig. 7A-C). The DH group also had significantly decreased occludin and claudin- 5 compared with the DM group $(P<0.05$; Fig. $6 \mathrm{~A}-\mathrm{C})$. Figure $7 \mathrm{~A}$ and $\mathrm{D}$ show that both the $\mathrm{DM}$ and $\mathrm{DH}$ groups had increased expression of the unstable matrix metalloproteinase MMP9, associated with pericyte function (NC vs. $\mathrm{DM}, P<0.05$, NC vs. DH, $P<0.05)$. The DH group had significantly higher MMP9 expression compared with the DM group $(P<0.05$; Fig. 7A, D). The DH group had significantly decreased PDGFR- $\beta$ and a-SMA expression compared with the NC and DM groups (PDGFR- $\beta$, NC vs. DH, $P<0.05$, DM vs. DH, $P<0.05$; aSMA, NC vs. DH, $P<0.01$, DM vs. DH, $P<0.05$; Fig. 7A, E, F).

\subsection{Severe hypoglycemia aggravated cognitive dysfunction}

Seven days after severe hypoglycemia, the mice was tested for sensorimotor deficits and spatial learning. Sensorimotor testing by the grip strength test showed no statistically significant difference in the duration of wire gripping between the three groups (NC, DM, DH: $12.2 \pm 2.1 \mathrm{~s}, 14.1 \pm 1.2 \mathrm{~s}, 13.1 \pm 1.3 \mathrm{~s}$, respectively, $P>0.05$; Fig. 8A). The effect of severe hypoglycemia on the learning ability of visuospatial memory in the diabetic mice was evaluated using the classical Morris water maze behavioral experiment. No significant difference was seen between the swimming speeds of the three groups $(P>0.05$, Fig. 8B). The escape latency (time spent searching for a hidden platform) gradually decreased as the training days increased (Fig. 8C). The DH group had significantly longer escape latency compared to the NC group $(P<0.01$, Fig. 8C); the DM group had prolonged escape latency compared to the NC group, but this was not significantly different $(P>0.05)$. In the spatial exploration experiment with the platform removed, the $\mathrm{DH}$ mice passed the original platform location significantly less often compared to the NC and DM mice $(P<$ 0.01 , Fig. 8D, E).

\section{Discussion}

The occurrence of hypoglycemia is common in the daily glucose management of diabetic patients, and a growing number of studies have suggested that hypoglycemia plays an important role in the pathogenesis of cognitive dysfunction in diabetic patients [19-21], but the underlying molecular 
mechanism remains unclear. In the present study, we demonstrated that severe hypoglycemia has a significant deleterious effect on cognitive function in diabetic mice. More importantly, we demonstrate for the first time that this effect may be associated with pericyte dysfunction and BBB disruption.

Glucose is the main source of human energy and cannot be synthesized and stored in the brain, so a continuous glucose supply to the brain is essential for maintaining cognitive function. Severe hypoglycemia can cause permanent neuronal damage and further structural damage to the brain, leading to altered cognitive function [9]. Previously, we found that mitochondrial homeostasis in hippocampal tissue was imbalanced after diabetic mice experienced repeated non-severe hypoglycemia, thereby causing cognitive impairment $[22,23]$. In the present study, our group continued our previous model of severe hypoglycemia in diabetic mice [18], in which a single injection of STZ caused absolute destruction of pancreatic islet $\beta$ cells and clinical manifestations of type 1 diabetes mellitus with obvious "excessive drinking, polyphagia, polyuria, and weight loss". To eliminate the influence of confounders such as physical strength and emotion of the mice after severe hypoglycemia on cognitive function testing, the mice that underwent the behavioral tests performed the grip test after a full 1-week rest. The results showed similar abilities in grip strength and swimming speed among the groups, with no measurable differences in sensorimotor coordination and strength between the groups, suggesting that the absence of gross motor impairment may affect the cognitive function of the mice. The Morris water maze subsequently demonstrated that severe hypoglycemia in the diabetic state can cause significant deficits in memory consolidation and working memory capacity in mice.

The BBB is a highly selective, semi-permeable border that separates circulating blood from extracellular fluid in the brain and central nervous system, and is formed by EC in the capillary wall, astrocyte ends wrapped around the capillaries, and pericytes embedded in the BM of the capillaries. Pericytes, a BBB component, share a BM with $\mathrm{EC}$, and in regions lacking a BM, the intersection of pericytes and $\mathrm{EC}$ membranes, termed a peg-rivet contact, forms a direct connection that controls molecular exchange between pericytes and EC [24]. Under physiological conditions, pericytes play an important role in BBB formation and maintenance, neurovascular system regulation, inflammatory cell transport, and toxic metabolite removal from the brain [25]. In recent years, the relationship between BBB function and AD has become a topic of interest. A study [26] showed that AD patients had greater BBB leakage compared to the healthy population, and the investigator concluded that BBB disruption is an early biomarker of cognitive dysfunction in humans. It has also been argued [27] that BBB integrity is lost prior to cognitive decline. Pericyte deficiency has been observed in animal models of $A D$ and in postmortem histological studies [28]. In another study [29], capillaries in the brain tissue of AD patients and in mice bred to develop $A D$ pathology were extruded by the pericytes and showed significant dysfunction. The authors calculated that this capillary constriction was sufficiently severe to halve blood flow, which is comparable to the reduction in blood flow to the part of the brain affected by $A D$, and they proposed that restoring perivascular cell function in the brain holds promise for treating AD. In conclusion, pericytes may play an important role in the pathogenesis of cognitive dysfunction. Therefore, is hypoglycemia-induced cognitive dysfunction associated with pericyte/BBB disruption? Studies have found that [25] hypoglycemia potentially damages BBB integrity and function, and hypoglycemia exposure severely affected the 
expression of BBB oxidation and inflammatory stress markers. However, there are no studies on BBB disruption in diabetic mice after severe hypoglycemia. In the present study, diabetic mice with severe hypoglycemia showed increased Evans blue infiltration and significant brain edema, suggesting severe BBB disruption. BBB/neurovascular unit impairment is now considered one of the key factors contributing to the development of diabetic encephalopathy [30], and diabetic mice exhibit enhanced BBB permeability after severe hypoglycemia, which may be associated with the loss of perivascular cells in the brain. To clarify the mechanism of BBB disruption, we examined the indicators related to pericyte function and distribution, and show that pericyte-specific expression of the proteins PDGFR- $\beta$ and a-SMA was reduced in the brains of diabetic mice after severe hypoglycemia, suggesting that the pericytes were lost. The transmission electron microscopic findings also suggest that diabetic mice have BBB disruption and pericyte damage after severe hypoglycemia. The preliminary results of the present study suggest that the mechanism of cognitive dysfunction after severe hypoglycemia in diabetic mice may be related to BBB disruption, which may result from pericyte dysfunction and loss. Therefore, how might pericytes in hypoglycemia affect BBB function?

The presence of oxidative stress during hypoglycemia has been well demonstrated, with increased reactive oxygen species (ROS) production in mitochondria isolated from the hippocampus of diabetic rats exposed to recurrent hypoglycemia [31]. In addition, elevated levels of inflammatory factors such as TNF- $a$ and IL- 6 have been observed in hypoglycemia, causing an acute inflammatory state in the organism [32]. MMPs are zinc-dependent proteases that degrade many structural components of the extracellular matrix and non-extracellular matrix proteins [13], and include MMP9. Oxidative stress and an inflammatory environment contribute to MMP9 activation and secretion by pericytes, which decreases the expression of TJ proteins and leads to BBB destruction [33]. MMP9 inhibitors reduce pericyte-associated BBB leakage [34]. In the present study, MMP9 expression was increased in diabetic mice and severely hypoglycemic mice, and the levels of occludin and claudin-5, closely related to BBB function, were further decreased; ultramicroscopy of mouse brain hippocampal tissue also showed significant TJ disruption. It is suggested that severe hypoglycemia in the high-glucose state can cause increased MMP9 expression in brain pericytes, which further causes degradation of the TJ proteins and leads to BBB disruption.

Notably, in the present study, although MMP9 levels were increased and TJ proteins were decreased in the diabetic state, no further BBB disruption or reduced pericyte numbers by hyperglycemia was observed, and the water maze behavior also suggested no significant cognitive dysfunction in the diabetic mice alone. Although diabetes is associated with increased risk of neurodegeneration and dementia [35], the specific effects of hyperglycemia on pericyte/BBB function remain controversial. Several studies have suggested [36] that the mechanism of pericyte dysfunction and consequent BBB leakage in diabetic patients is related to enhanced ROS and reduced ATP production due to oxidative stress caused by prolonged hyperglycemia. In contrast, others have found that BBB function remained unchanged in diabetes [15]. Yet, our results show that hyperglycemia alone caused a slight impairment in pericyte function and in BBB morphology and function compared to that in normal mice. However, it was insufficient to cause significant BBB disruption and subsequent cognitive dysfunction as compared to the severe hypoglycemia group. We conjecture that the possible reason for the high glucose in this model is 
the short duration of the study (only 5 days from sampling and 12 days from the water maze test), and the glycosylation products and oxidative stress of the high glucose were insufficient to cause serious BBB disruption and thereby lead to cognitive dysfunction. However, according to the trend of our results, it is reasonable to think that long-term hyperglycemia will destroy pericyte/BBB function. To explore the effect of hyperglycemia on pericyte/BBB function, further investigations can extend the duration of hyperglycemia in diabetic mice or use diabetic genotype mice.

It is well known that neuronal damage is a key factor in cognitive dysfunction [37]. The loss of pericytes may disrupt the BBB through the leakage and deposition of vascular and neurotoxic macromolecules from peripheral circulation sources. The associated microvascular degeneration and edema may cause chronic hypoxia by further increasing the entry of neurotoxic substances and causing reduced blood flow, resulting in structural and functional changes in neurons, thereby affecting interneuronal interconnections and ultimately exacerbating the development of primary neurodegenerative disease [38]. Pericyte-deficient mice have increased neuronal apoptosis, reduced neuronal numbers, and behavioral deficits in the cortex and hippocampus [39]. In the present study, HE staining revealed significant neuronal destruction in the severely hypoglycemic mice, and we speculate that the cause may be the leakage and deposition of vascular and neurotoxic macromolecules after pericyte/BBB dysfunction that further exacerbate neuronal damage, thereby causing cognitive dysfunction. The specific molecular mechanism requires further research.

This study has some limitations. First, this study used STZ injection to create a diabetic mice model, and it was reported that STZ injection could directly cause brain damage [40]. However, in the present study, mice in the group that experienced severe hypoglycemia had significantly more impairment in cognitive function and BBB compared with mice in the diabetic group that received STZ injection alone, suggesting an independent or additive effect of hypoglycemia on these impairments. Second, the effect of severe hypoglycemia on pericytes/the BBB has not been further explored at the cellular level, and the specific mechanisms have not been explored in depth. Our study shows, for the first time, that the severe hypoglycemia-induced cognitive dysfunction in diabetic mice is associated with pericyte/BBB dysfunction.

\section{Conclusion}

Our results suggest that severe hypoglycemia in diabetic mice can lead to the development of cognitive dysfunction. The possible mechanism is that severe hypoglycemia occurs under diabetes, which leads to pericyte dysfunction and subsequently BBB destruction. Our experimental results will aid the elucidation of the role of severe hypoglycemia and cognitive dysfunction, and also contribute to providing new biomarkers for early prediction of the development of cognitive impairment due to severe hypoglycemia.

\section{Declarations}

\section{Ethics approval and consent to participate}


All experiments were approved by the Institutional Animal Care and Use Committee of Fujian Medical University (Approval No. FJMU IACUC 2018-060) and complied with the National Institutes of Health guide for the care and use of Laboratory animals (NIH Publications No. 8023, revised 1978).

\section{Consent for publication}

Not applicable

\section{Availability of data and materials}

The datasets used during the current study are available from the corresponding author on reasonable request.

\section{Competing interests}

The authors declare that they have no competing interests.

\section{Funding}

This work was supported by the Fujian Science and Technology Innovation Joint Fund Project (2017Y9060), the Joint Funds for the innovation of science and Technology, Fujian province (2019Y9062), and the Startup Fund for Scientific Research of Fujian Medical University (2020QH2022).

\section{Authors' contributions}

L Lin contributed to the conception of the study and manuscript. LJ Wang performed the experiments and analysed the data. LS Huang contributed to perform the experiments. YB Wu contributed to perform the experiments. $Z$ Chen contributed to the conception of the study and manuscript preparation. LB Liu contributed to the conception of the study and manuscript preparation.

\section{Acknowledgements}

The authors acknowledge the support provided by National Natural Science Foundation of China and Joint Funds for the innovation of Science and Technology, Fujian Province.

\section{References}

1. International Diabetes Federation. Diabetes Atlas 9th edition, 2019, IDF, Brussels. Available at: https://diabetesatlas.

2. Monnier L, Colette $\mathrm{C}$, Owens $\mathrm{D}$. The glycemic triumvirate and diabetic complications: is the whole greater than the sum of its component parts? Diabetes research and clinical practice 2012; 95:303311. 
3. Srikanth Velandai,Sinclair Alan J, Hill-Briggs, Felicia, et al. Type 2 diabetes and cognitive dysfunctiontowards effective management of both comorbidities.[J].Lancet Diabetes Endocrinol, 2020, 8: 535545.

4. van Sloten Thomas T, Sedaghat Sanaz,Carnethon Mercedes R, et al. Cerebral microvascular complications of type 2 diabetes: stroke, cognitive dysfunction, and depression.[J]. Lancet Diabetes Endocrinol. 2020;8:325-36.

5. Biessels Geert Jan,Nobili Flavio,Teunissen. Charlotte E, et al. Understanding multifactorial brain changes in type 2 diabetes: a biomarker perspective.[J].Lancet Neurol, 2020, 19: 699-710.

6. Kullmann Stephanie,Kleinridders André,Small. Dana M, et al. Central nervous pathways of insulin action in the control of metabolism and food intake.[J]Lancet Diabetes Endocrinol, 2020, 8: 524-34.

7. Lee AK, Rawlings AM, Lee CJ, Gross AL, Huang ES, Sharrett AR, et al. Severe hypoglycaemia, mild cognitive impairment, dementia and brain volumes in older adults with type 2 diabetes: the atherosclerosis risk in communities (ARIC) cohort study. [J] Diabetologia. 2018;61:1956-65.

8. Abdelhafiz AH, Sinclair AJ. Cognitive Frailty in Older People with Type 2 Diabetes Mellitus: the Central Role of Hypoglycaemia and the Need for Prevention.[J]. Curr Diab Rep. 2019;19:15.

9. Jackson David A,Michael Trevin,Vieira de Abreu Adriana et al. Prevention of Severe HypoglycemiaInduced Brain Damage and Cognitive Impairment With Verapamil.[J].Diabetes, 2018, 67: 2107-2112.

10. Sweeney Melanie D, Sagare Abhay P, Zlokovic Berislav V. Blood-brain barrier breakdown in Alzheimer disease and other neurodegenerative disorders.[J]. Nat Rev Neurol. 2018;14:133-50.

11. Montagne Axel,Nation Daniel A, Sagare Abhay P, et al. APOE4 leads to blood-brain barrier dysfunction predicting cognitive decline[J]Nature, 2020, 581: 71-6.

12. Nakazaki M, Sasaki M, Kataoka-Sasaki Y, et al. Intravenous Infusion of Mesenchymal Stem Cells Improves Impaired Cognitive Function in a Cerebral Small Vessel Disease Model[J]. Neuroscience. 2019;408:316-37.

13. Hawkins BT, Lundeen TF, Norwood KM, et al. Increased blood-brain barrier permeability and altered tight junctions in experimental diabetes in the rat: contribution of hyperglycaemia and matrix metalloproteinases. [J]Diabetologia. 2007;50:202-11.

14. Janelidze Shorena,Hertze Joakim,Nägga. Katarina, et al. Increased blood-brain barrier permeability is associated with dementia and diabetes but not amyloid pathology or APOE genotype.[J]Neurobiol Aging, 2017, 51: 104-12.

15. Mäe MA,Li Tian,Bertuzzi Giacomo, et al. Prolonged systemic hyperglycemia does not cause pericyte loss and permeability at the mouse blood-brain barrier.[J]Sci Rep, 2018, 8: 17462.

16. Yorulmaz Hatice,Seker Fatma Burcu. Oztas Baria,The effects of hypoglycemic and alcoholic coma on the blood-brain barrier permeability.[. J]Bosn J Basic Med Sci. 2011;11:108-12.

17. Zhao Fei,Deng Jiangshan,Xu Xiaofeng. et al. Aquaporin-4 deletion ameliorates hypoglycemiainduced BBB permeability by inhibiting inflammatory responses.[. J]J Neuroinflammation. 2018;15:157. 
18. Huang L,Zhou Yu,Chen Zhou, et al. Severe hypoglycemia exacerbates myocardial dysfunction and metabolic remodeling in diabetic mice.[J]Mol Cell Endocrinol, 2020, 503: 110692.

19. Bree Adam J, Puente Erwin C, Daphna-Iken, Dorit, et al. Diabetes increases brain damage caused by severe hypoglycemia.[. J]Am J Physiol Endocrinol Metab. 2009;297:E194-201.

DOI:10.1152/ajpendo.91041.2008.

20. Whitmer Rachel A, Karter Andrew J, Yaffe Kristine, et al. Hypoglycemic episodes and risk of dementia in older patients with type 2 diabetes mellitus.[J].JAMA, 2009, 301: 1565-72.

21. Chin Sang Ouk,Rhee Sang Youl,Chon Suk. et al. Hypoglycemia is associated with dementia in elderly patients with type 2 diabetes mellitus: An analysis based on the Korea National Diabetes Program Cohort.[J]. Diabetes Res Clin Pract. 2016;122:54-61.

22. Yu Z, Lishan H, Wenting Z, et al. Recurrent non-severe hypoglycemia exacerbates imbalance of mitochondrial homeostasis leading to synapse injury and cognitive deficit in diabetes[J]. American Journal of Physiology Endocrinology Metabolism. 2018;315:E973-86.

23. Zhou Yu,Lian Siheng,Zhang Jin, et al. Mitochondrial Perturbation Contributing to Cognitive Decline in Streptozotocin-Induced Type 1 Diabetic Rats[J]Cell Physiol Biochem, 2018, 46: 1668-82.

24. Hort, Jakub,Vališ Martin,Kuča Kamil et al. Vascular Cognitive Impairment: Information from Animal Models on the Pathogenic Mechanisms of Cognitive Deficits.[J].Int J Mol Sci, 2019, 20: undefined.

25. Uemura Maiko T,Maki Takakuni,Ihara Masafumi, et al. Brain Microvascular Pericytes in Vascular Cognitive Impairment Dementia[J]Front Aging Neurosci, 2020, 12: 80.

26. van de Haar Harm J,Burgmans, Saartje,Jansen Jacobus FA, et al. Blood-Brain Barrier Leakage in Patients with Early Alzheimer Disease[J]Radiology. 2016;281:527-35.

27. Blanchard Joel W, Bula Michael,Davila-Velderrain Jose. et al. Reconstruction of the human bloodbrain barrier in vitro reveals a pathogenic mechanism of APOE4 in pericytes. [J]Nat Med. 2020;26:952-63.

28. Nikolakopoulou AM, Zhao Z, Montagne A, Zlokovic BV. Regional early and progressive loss of brain pericytes but not vascular smooth muscle cells in adult mice with disrupted platelet-derived growth factor receptor- $\beta$ signaling. PLoS ONE. 2017;12:e0176225.

29. Nortley Ross,Korte Nils,Izquierdo Pablo. et al. Amyloid $\beta$ oligomers constrict human capillaries in Alzheimer's disease via signaling to pericytes. [J]Science. 2019;365:undefined.

30. Bogush Marina. Heldt Nathan A,Persidsky Yuri,Blood Brain Barrier Injury in Diabetes: Unrecognized Effects on Brain and Cognition.[. J]J Neuroimmune Pharmacol. 2017;12:593-601.

31. Shukla Vibha,Fuchs Perry,Liu Allen et al. Recurrent Hypoglycemia Exacerbates Cerebral Ischemic Damage in Diabetic Rats via Enhanced Post-Ischemic Mitochondrial Dysfunction[J]Transl Stroke Res, 2019, 10: 78-90.

32. Solis-Herrera Carolina,Sheikh Omar,Chilton Robert,A new perspective on lowering CV risk from hypoglycaemia.[J].Eur Heart J, 2020, 41: 218-220. 
33. Persidsky Yuri,Hill Jeremy,Zhang Ming. et al. Dysfunction of brain pericytes in chronic neuroinflammation.[J]. J Cereb Blood Flow Metab. 2016;36:794-807.

34. Underly Robert G,Levy Manuel,Hartmann David A et al. Pericytes as Inducers of Rapid, Matrix Metalloproteinase-9-Dependent Capillary Damage during Ischemia.[J].J Neurosci, 2017, 37: 129140.

35. Biessels Geert Jan,Staekenborg Salka,Brunner Eric. et al. Risk of dementia in diabetes mellitus: a systematic review.[. J]Lancet Neurol. 2006;5:64-74.

36. Liu Yedan,Zhang Huawei,Wang Shaoxun et al. Reduced pericyte and tight junction coverage in old diabetic rats are associated with hyperglycemia-induced cerebrovascular pericyte dysfunction.[J].Am J Physiol Heart Circ Physiol. 2020, undefined: undefined.

37. Tobin Matthew K,Musaraca Kianna,Disouky Ahmed, et al. Human Hippocampal Neurogenesis Persists in Aged Adults and Alzheimer's Disease Patients.[J]Cell Stem Cell, 2019, 24: 974-82.e3.

38. Nikolakopoulou Angeliki M,Montagne Axel,Kisler Kassandra, et al. Pericyte loss leads to circulatory failure and pleiotrophin depletion causing neuron loss.[J]Nat Neurosci, 2019, 22: 1089-98.

39. Kisler Kassandra,Nelson Amy R, Rege Sanket V, et al. Pericyte degeneration leads to neurovascular uncoupling and limits oxygen supply to brain.[J].Nat Neurosci, 2017, 20: 406-416.

40. Biswas Joyshree,Gupta Sonam,Verma Dinesh Kumar. et al. Streptozotocin alters glucose transport, connexin expression and endoplasmic reticulum functions in neurons and astrocytes.

[J].Neuroscience, 2017, 356: 151-166.

\section{Figures}




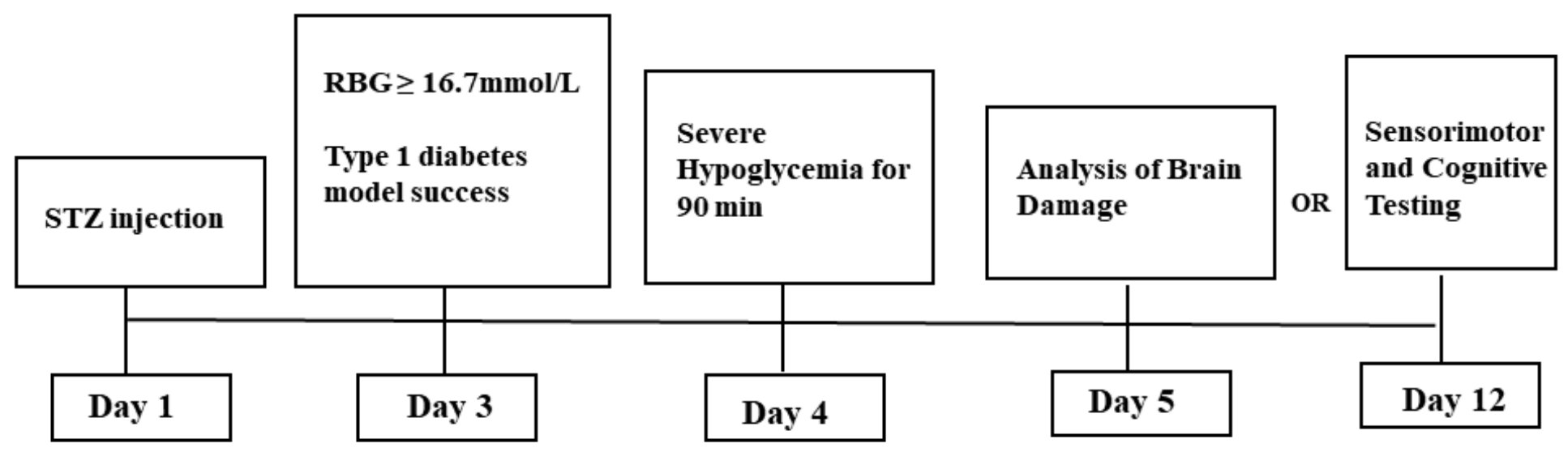

Figure 1

Experimental protocol. 

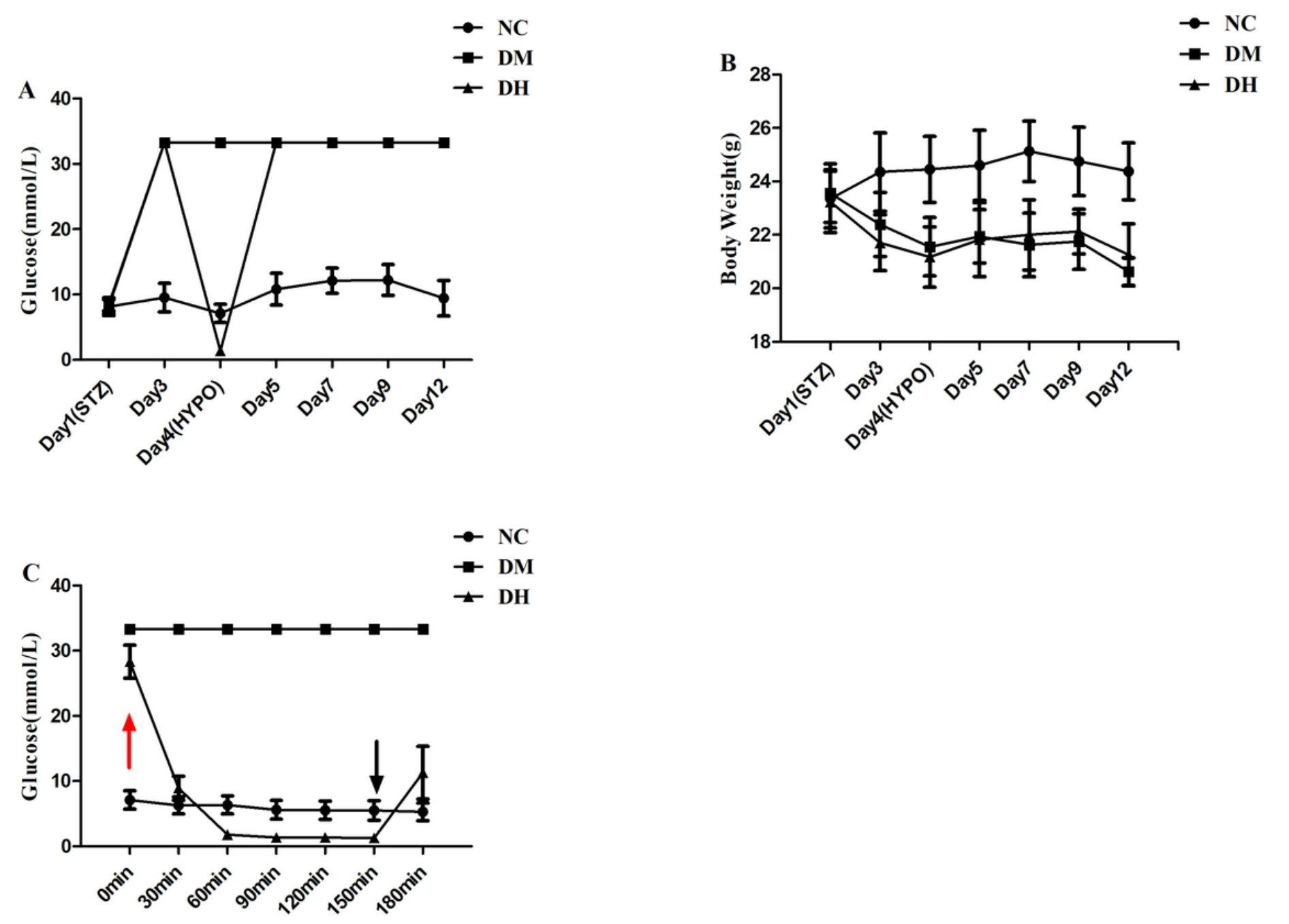

Figure 2

Blood glucose and body weight data. (A) Glycemia levels of the three groups. (B) Body weight trajectory of the three groups. (C) Glucose levels of the three groups during the severe hypoglycemia episode $(<2.0$ $\mathrm{mmol} / \mathrm{L})$. NC, normal control; DM, type 1 diabetes mellitus; $\mathrm{DH}$, severe hypoglycemia. Data are the means \pm SEM. 


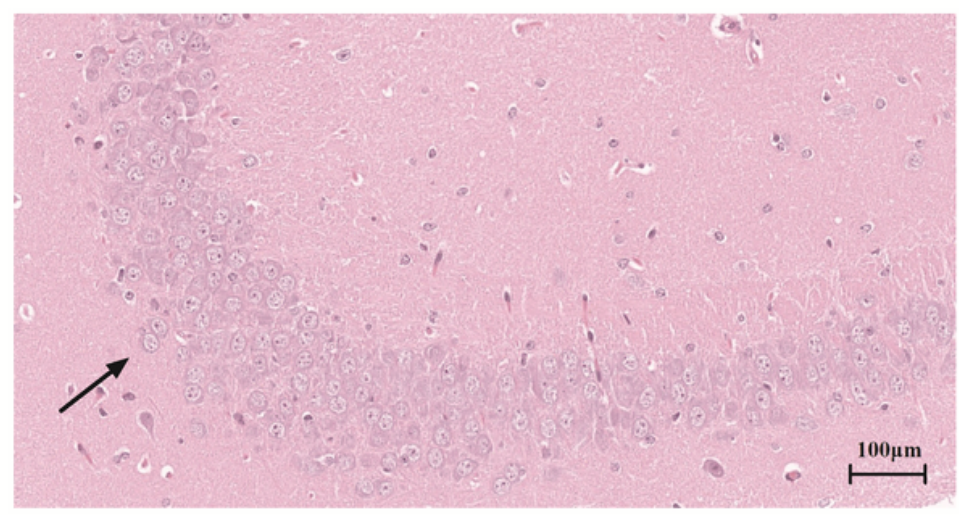

NC

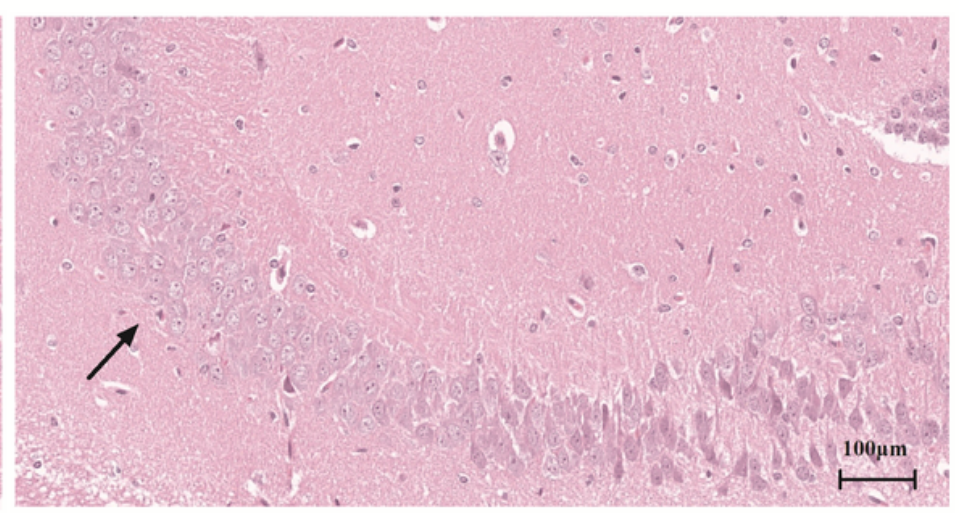

DM

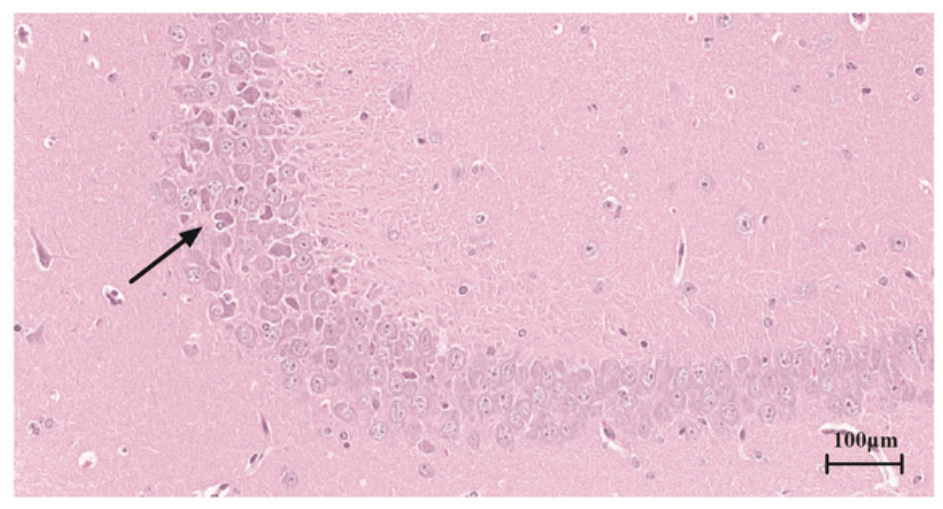

DH

\section{Figure 3}

Severe hypoglycemia under diabetic conditions causes histological changes in the hippocampal region. The black arrowheads indicate layers of pyramidal cells. Scale bar $=100 \mu \mathrm{m}$ (HE staining).

A

NC

DM

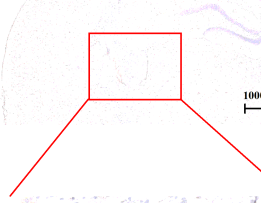

$=$

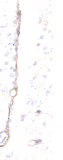

iomen
DH

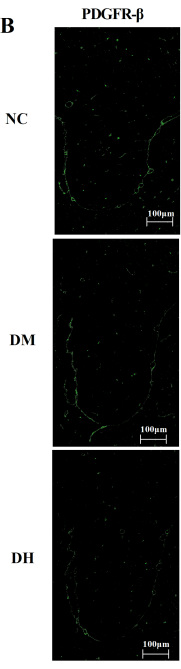

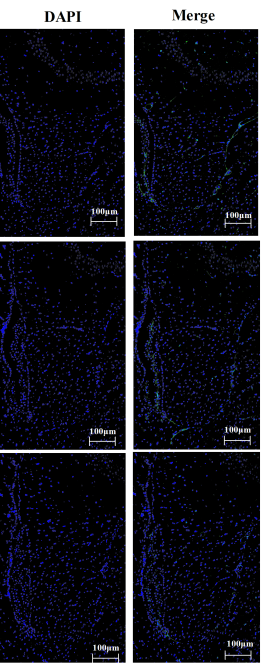
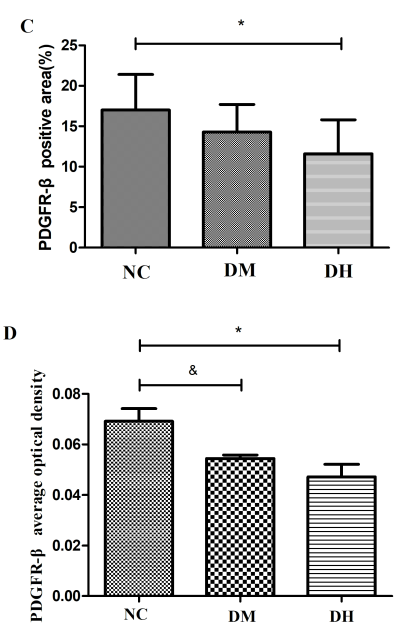

Figure 4

Effect of severe hypoglycemia on PDGFR- $\beta$ expression in diabetic mouse hippocampus. (A) Representative images of immunohistochemical photographs of PDGFR- $\beta$ in the brain. (B) Quantitation 
of PDGFR- $\beta$ accumulation. (C) PDGFR- $\beta$ positive expression is observed as green fluorescence; DAPIstained nuclei are blue under UV excitation. (D) Comparison of quantitative analysis of mean optical density in the mice with positive PDGFR- $\beta$ expression in brain capillary mid-peripheral cells in the hippocampus. Scale bar $=100 \mu \mathrm{m}$. Data are the means \pm SEM. \&P $<0.05 \mathrm{NC}$ vs. $D M,{ }^{*} P<0.05 \mathrm{NC}$ vs. DH.

NC

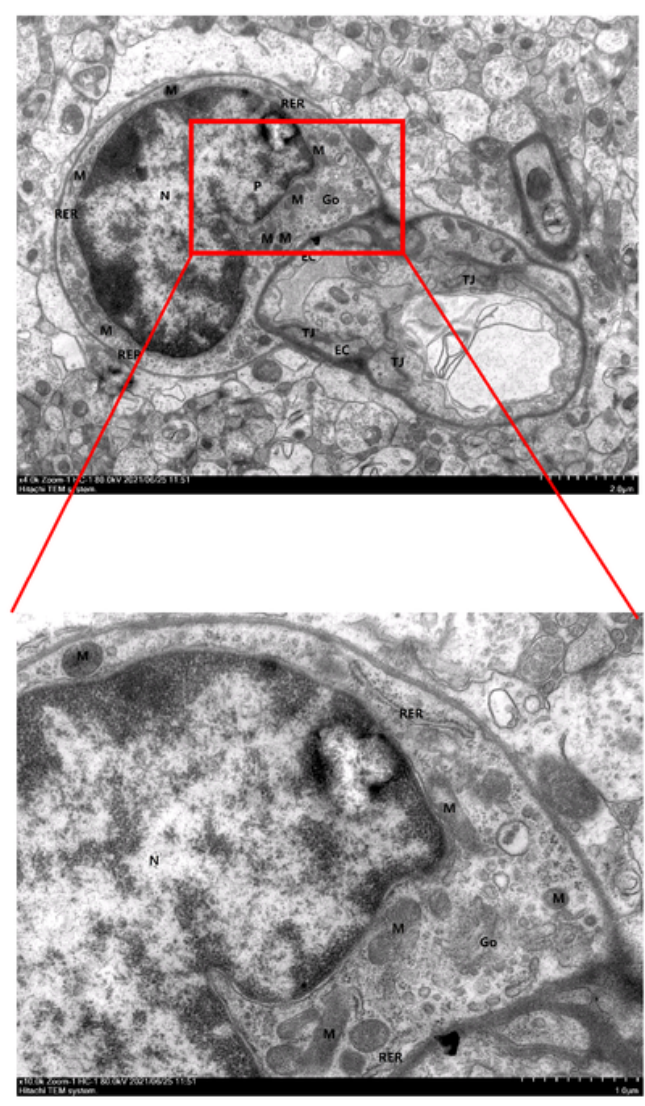

DM

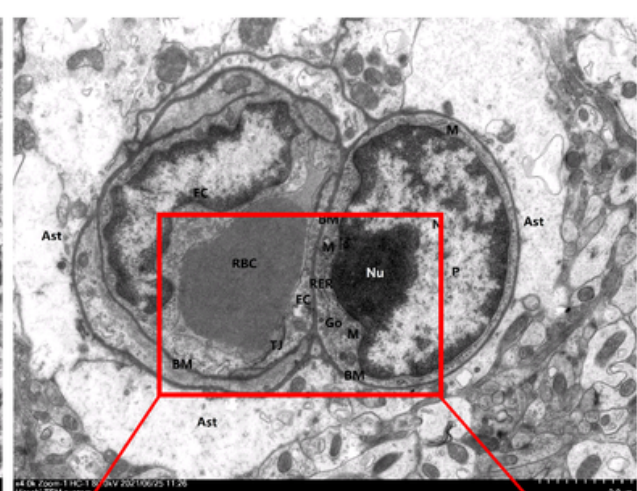

\section{DH}

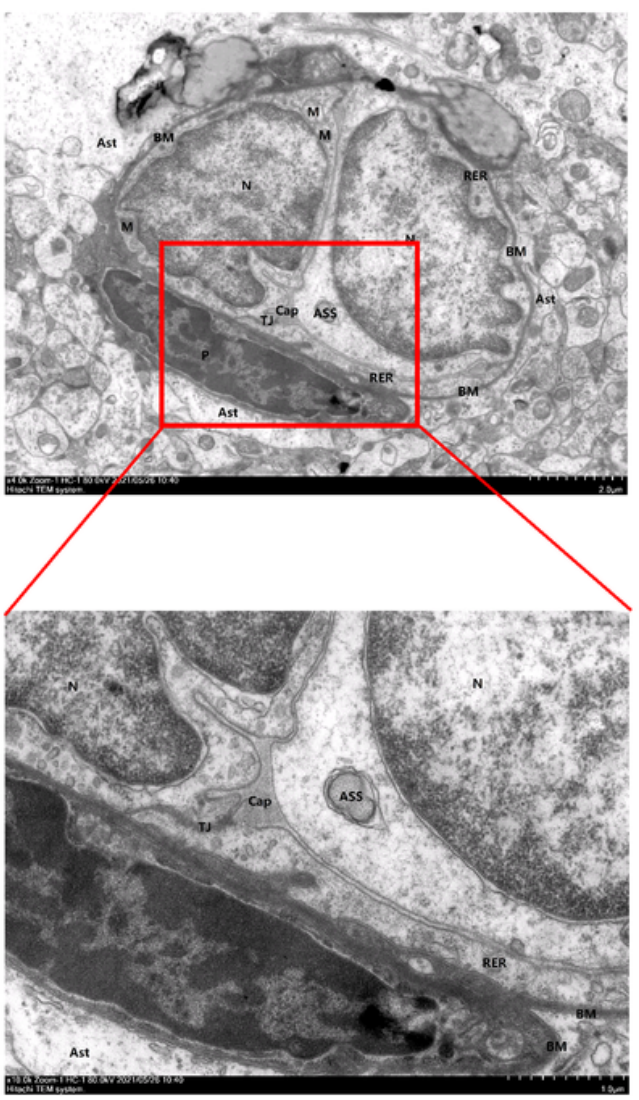

Figure 5

Severe hypoglycemia aggravated BBB structural damage and pericyte damage. Representative images of hippocampal BBB and pericyte morphology under transmission electron microscopy are shown. Ast, astrocyte; P, pericyte; N, nucleus; Nu, nucleolus; Go, Golgi apparatus. 
A

NC
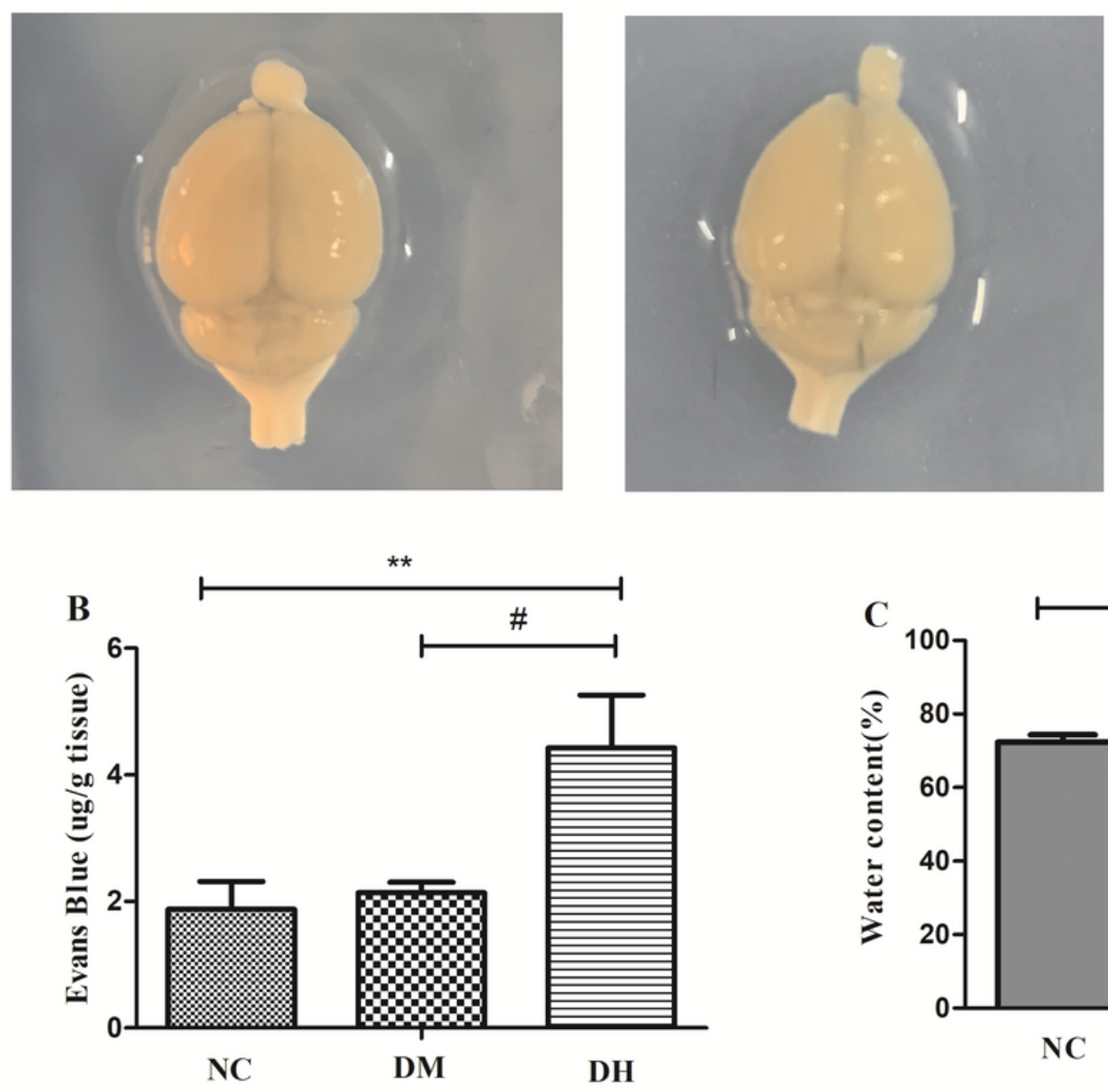

DM
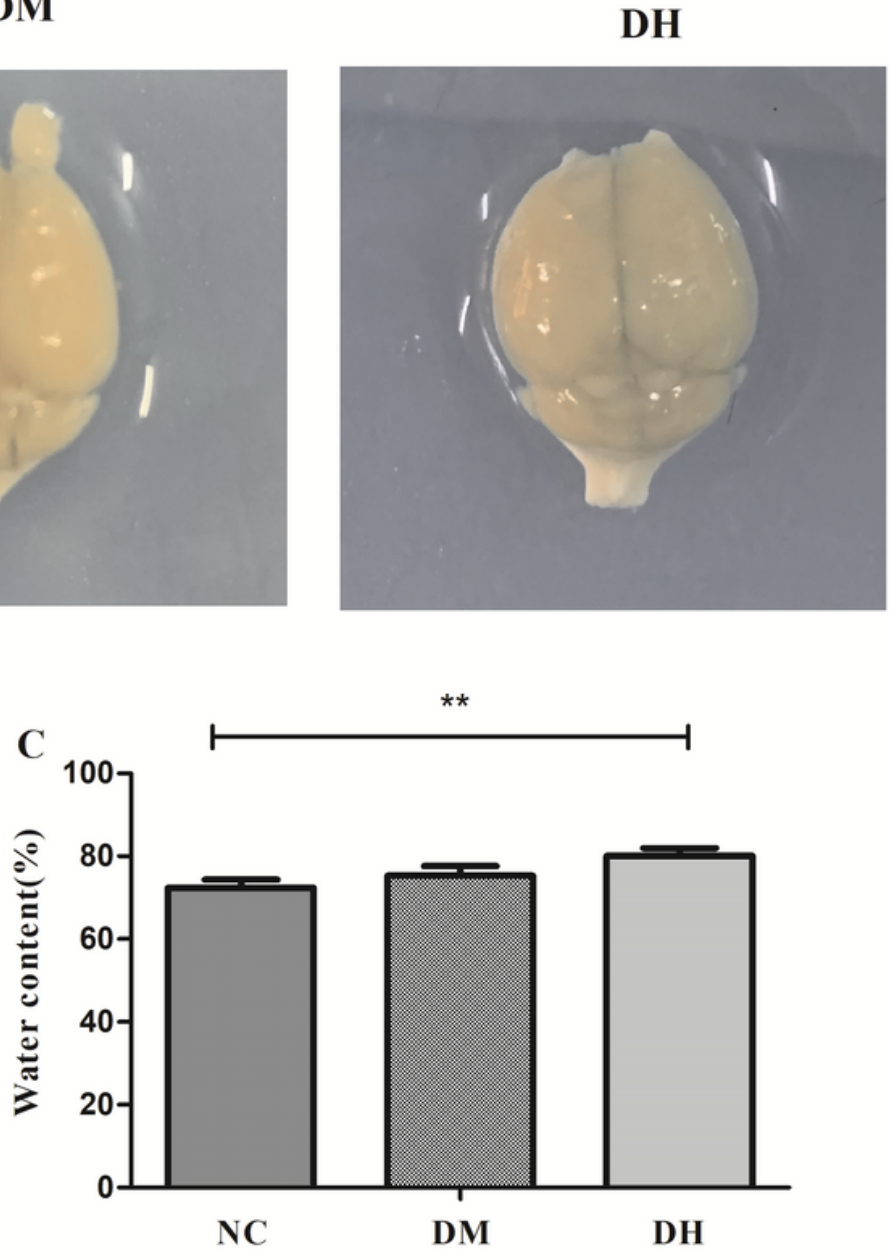

\section{Figure 6}

Effects of severe hypoglycemia on BBB and brain edema. (A) Naked-eye view of Evans blue exocytosis in mouse brain. (B) Quantification of Evans blue extravasation. (C) Mouse brain water content. Data are the means \pm SEM. ${ }^{\star \star P}<0.01 \mathrm{NC}$ vs. $\mathrm{DH}, \& \mathrm{P}<0.05 \mathrm{NC}$ vs. $\mathrm{DM}, \# \mathrm{P}<0.05 \mathrm{DM}$ vs. $\mathrm{DH}$. 
A

Occludin

Claudin 5

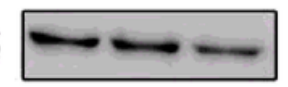

MMP9

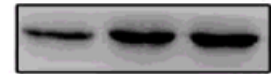

PDGFR- $\beta$

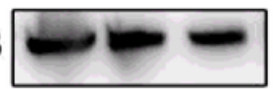

$\beta$-actin

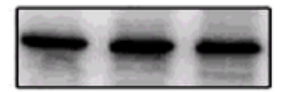

$\alpha$-SMA

B

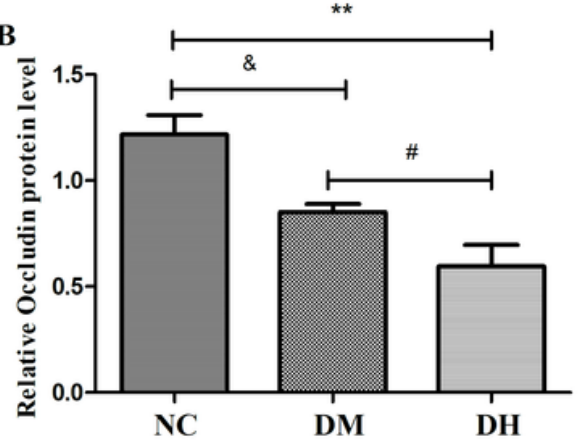

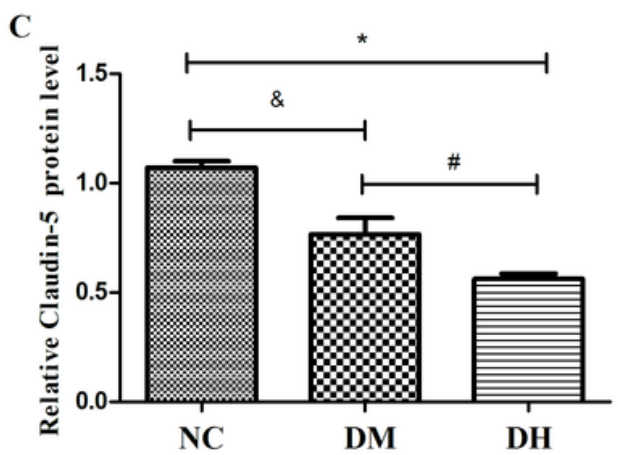

GAPDH
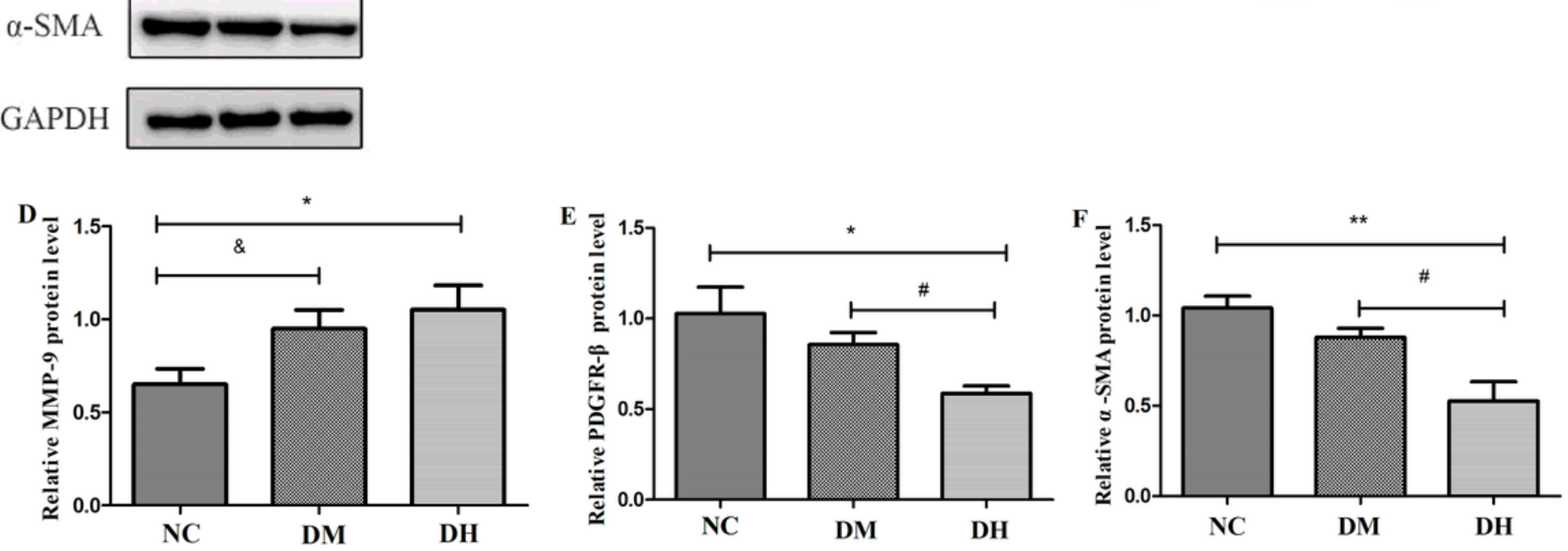

Figure 8

Severe hypoglycemia caused reduced expression of BBB TJ binding proteins and pericyte-specific proteins. (A) Representative immunoblot images of relative protein expression. Western blot analysis was performed for the protein levels of occludin (B), claudin-5 (C), MMP9 (D), PDGFR- $\beta$ (E), and a-SMA (F). Data are the means \pm SEM. ${ }^{\star *} \mathrm{P}<0.01 \mathrm{NC}$ vs. $\mathrm{DH},{ }^{*} \mathrm{P}<0.05 \mathrm{NC}$ vs. $\mathrm{DH}, \# \mathrm{P}<0.05 \mathrm{DM}$ vs. $\mathrm{DH}$. 

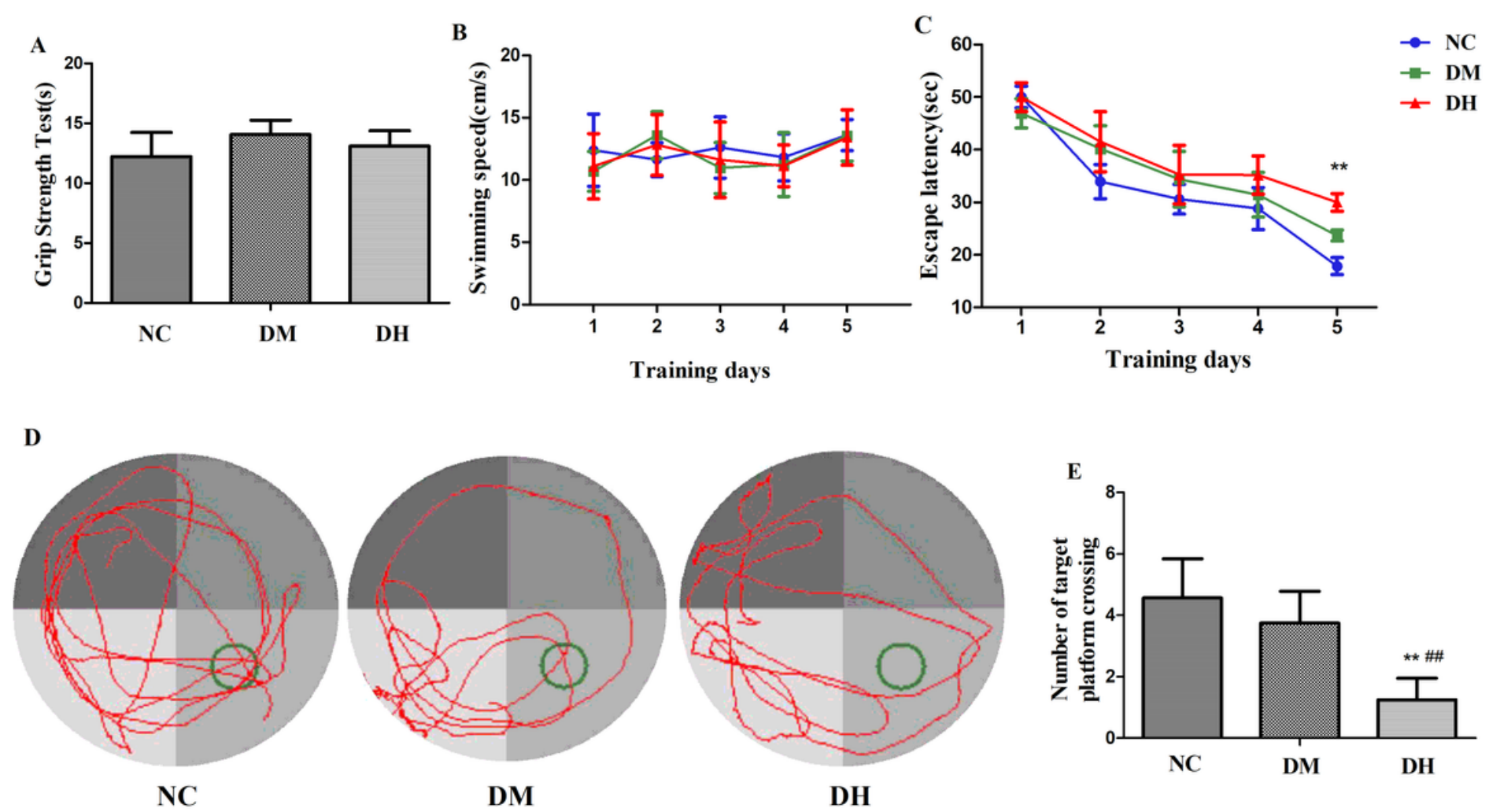

Figure 9

Severe hypoglycemia caused cognitive dysfunction. (A) Sensorimotor testing by grip strength test. (B) Escape latency. (C) Swimming speed. (D) Representative traces of swimming paths of each group in the probe test. $(E)$ Crossings of the target quadrant during the probe trial. $n=10$ per group. Data are the means \pm SEM. ${ }^{*} \mathrm{P}<0.01 \mathrm{NC}$ vs. $\mathrm{DH}, \# \# \mathrm{P}<0.01 \mathrm{DM}$ vs. $\mathrm{DH}$. 